\title{
Avifaunal diversity in relation to vegetation height and coverage in grasslands of Shuklaphanta Wildlife Reserve
}

\author{
S. Dhoubhadel ${ }^{*}$ \\ Central Department of Environment Science, Tribhuvan University, Kathmandu, Nepal
}

\begin{abstract}
The grasslands of Nepal's Terai are extremely rich in bird species with several globally threatened species. These birds are often quite sensitive to changes in the habitat quality and microclimate around them making them ideal indicators of the grassland ecosystem health. Unfortunately, very little is known about the grassland avifauna. This study was conducted to determine the avifaunal composition and diversity with respect to the vegetation height and patch openness in the grasslands of Shuklaphanta Wildlife Reserve by using open width line transect method along fire-lines. The birds and the vegetation were recorded using BBIRD protocol and the species diversity was calculated. The Kruskal-Walli test for differences in median populations and Canonical Correspondence Analysis (CCA) were also conducted. This study recorded 104 species birds belonging to 39 families among which 29 species were insectivorous, 17 herbivorous, 17 carnivorous and the rest omnivorous with 94 resident species and 10 summer visitor species. Covered patches of short grassland had highest number of species while covered patches of tall grasslands had the highest species diversity. The results from KruskallWalli test and CCA highlighted the need for the maintenance of ecological integrity of all the grassland habitat areas.
\end{abstract}

Key words: BBIRD, Birds, Canonical correspondence, Guild, Kruskal-Walli

\section{Introduction}

Birds play a major role in the functioning of many ecosystems, particularly through pollination and seed dispersal and are often quite sensitive to changes in the habitat quality and microclimate around them making them ideal indicators of the ecosystem health (BCN, 2005). They are also quite attractive and can be an excellent means to create awareness of nature and the environment (BCN, 2005).

Nepal is extremely rich in avifauna with about 863 bird species, 9.4 percent of the world's known birds (Shrestha and Gupta, 1993 , in BCN, 2005). The grasslands of lowland Nepal is the extremely rich in bird species compared to grassland elsewhere in the world (Tucker, 1991, in BCN, 2005) despite the fact most of them are now confined to the protected areas only. Many bird species that occur in these grasslands are globally threatened which includes almost all of the globally threatened birds that breed in Nepal (Baral, 2001). Despite this, the list of protected animals in Nepal lists only two grassland dependent bird species. For the revision of the list, the current

*Corresponding author, email address: dhoubhadel@gmail.com status and distribution of grassland birds and the updated checklist of the avifauna is necessary. While, most mammals that inhibit lowland grasslands are well studied (Dhungel \& O'Gara 1985, in BCN, 2005), and several reports have been published about Nepalese avifauna, studies on purely grassland dependent species apart from a few endangered species are rare. There is an urgent need for a greater understanding of status, distribution, ecology and habitat of grassland birds (Inskipp \& Inskipp, 1991).

This study was conducted to assess the avifaunal assemblages that have colonized Shuklaphanta Wildlife Reserve since the protected area has one of the extensive Terai grasslands in Nepal with many endangered bird species. The specific objectives of this research were to determine the avifaunal composition and diversity in the grasslands with respect to grassland vegetation type (dominant species average height) and patch openness (coverage removed due to grass cutting/fire). However, this study was more exploratory type and recorded the composition and diversity of only diurnal birds that are either resident or visitors during summer season. 


\section{Materials and Methods}

This study was conducted in April 2011 in the grassland sites namely, Shuklaphanta, Shikariphanta, Hariyaphanta, Khariyaphanta, Suyraphanta, Pipariyaphanta, Sundariphanta, Barkaule, Dudhiya, Mangalsera, Andhaiya, and Radhapur within Shuklaphanta Wildilfe Reserve. The grasslands can be divided into four types according to the average maximum height of dominant vegetation/grass species. They are:

1. Tall grassland consisting of Narenga, Saccharum and Themeda with Phragmites (height 2-5 m), 2. Short grassland of mainly Imperata (height $0.5-2 \mathrm{~m}$ ), 3. Very short grasslands of Cyanodon dactylon (height 0.25-0.75 m); and 4. Wooded grasslands which has trees shading the grasses (height $>5$ $\mathrm{m})$. For this study, the very short grasslands were included within the short grasslands.

The bird survey was conducted using open width line transect method along fire-lines. The birds were surveyed during clear weathered mornings (5:30 am to 10:30 am) and evenings (5:00 pm to $6: 30 \mathrm{pm}$ ), when birds are most active, using a pair of binoculars (OLYMPUS, 8-16X 40), and "Nepalese Birds" by Grimeett et al. (2003) for birds identification. The birds observed, the GPS coordinates, dominant/common vegetation composition of the area, vegetation cover (occular), vegetation height (using wein's pole), habitat type, weather, and time were recorded at every $500 \mathrm{~m}$ interval along transects using BBIRD protocol. The points along the transect was covered with the help of a land rover moving at a constant speed of $10 \mathrm{~km} / \mathrm{hr}$, and using the roof of the vehicle as a vantage point. Five minutes was spent at every specific points for bird observation. The points with $50 \%$ or more plant coverage was taken as covered patches, while those with less than $50 \%$ plant coverage were taken as open patches.

The species diversity, evenness and similarity between the different vegetation types and patchiness were calculated using Shanon-Weiner Diversity Index, Pielou's Evenness Index, and Sorenson's Similarity Index. The Kruskal-Walli test was used to test the equality of population medians of birds among different vegetation/patchiness type and the Canonical Correspondence Analysis (CCA) method was used to explain the effect of vegetation type and patchiness on avian diversity (using 7 most abundant species). The data analysis was made with the help of computer softwares: MS EXCEL, SPSS and PC-ORD.

\section{Results}

The study found that the grasslands of Shuklaphanta Wildlife Reserve had a high avian diversity with a total 104 species of birds belonging to 39 families and 15 orders. Among the species recorded, conservation status of 3 species was vulnerable, 1 species was threatened and 4 critically endangered according to IUCN Red Data Book. Out of total, 94 species were found to be resident species, while 10 species were found to be spring visitors.

The study also found that the birds could be categorized into four major guilds with 29 purely insectivorous species, 17 purely herbivorous species (grass, fruits, seeds and grains), 17 carnivorous species (other than insects), and 41 omnivorous species.

The total area was determined to be $49.02 \mathrm{~km}^{2}, 23.43 \mathrm{~km}^{2}$ and $7.12 \mathrm{~km}^{2}$ for short grassland, tall grassland and wooded grassland, respectively. The total number of species recorded was 52,39 , and 30 species for short grassland, tall grassland and wooded grassland, respectively. The total numbers of species recorded in open patches were 9,16 , and 5 while in covered patches were 38,42 and 27 , respectively for tall, short and wooded grasslands. Similarly, a total of 27 species were recorded in open patches, while 90 species were recorded in covered patches.

The avian diversity in tall, short and wooded grasslands was found to be $0.1597,0.1505$ and 0.0868 , respectively. The species diversity in covered patches were $0.1598,0.1590$ and 0.1520 , while in open patches were $0.0920,0.1251$ and 0.0634 , respectively for tall, short and wooded grasslands. The species diversity in open patches was 0.0011 , while in covered patches was 0.0153 . Passer domesticus (house sparrow) was found to be the most abundant species.

The tall and short grasslands were found to be $19.78 \%$, while the tall and wooded grasslands were found to be only $1.45 \%$ similar, and wooded and short grasslands were found to be 12.95\% similar. The open and closed patches were 22.22\% similar. The open and closed patches of tall grasslands were $25.53 \%$ similar, compared to $10.35 \%$ of short grasslands and $12.50 \%$ of wooded grasslands. 


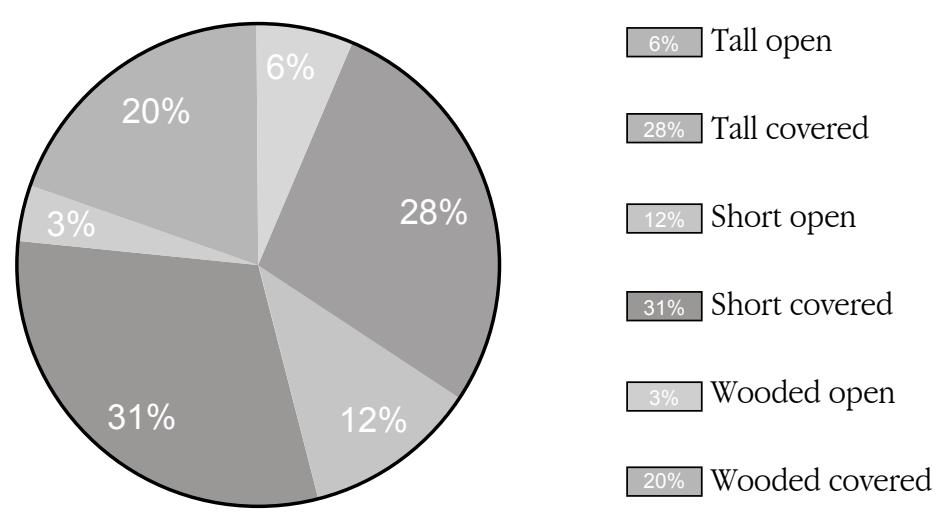

Fig. 1 Habitat preference of the birds recorded

The most preferred habitat by birds was covered patches of short grasslands (30\%) followed by covered patches of tall grasslands (27\%) (Fig. 1).

The Kruskall-Walli test conducted found the values of $K$ to be 0.45 for tall grasslands, 2.2 for short grasslands and 0.13 for wooded grasslands which showed that there was greater equality of population medians of birds in short grassland compared to tall and wooded grasslands.

The Canonical Correspondence Analysis of 7 most abundant bird species showed that the total number of individuals of each bird species decreased with increasing openness of plant cover which meant that the species preferred covered patches over open patches. In case of the relation between the numbers of individuals of the bird species to the height of the vegetation, there seems to be less pronounced relationship. The Nyctyornis athertoni (Na), Merops orientalis (Mo), and Apus affinis (Aa) was found to prefer vegetation with shorter height (short grasslands) while Merops philippinus (Mp), Cypsiurus balasiensis (Cb), Priania inornata (Pi) and Priania socialis (Ps) were found to prefer vegetation with median height (tall grassland). Nyctyornis atbertoni (Na), and Merops philippinus (Mp) was also found to prefer quite tall vegetation (wooded grassland). All 7 species abundance was significantly associated with the two environmental variables denoted in axis.

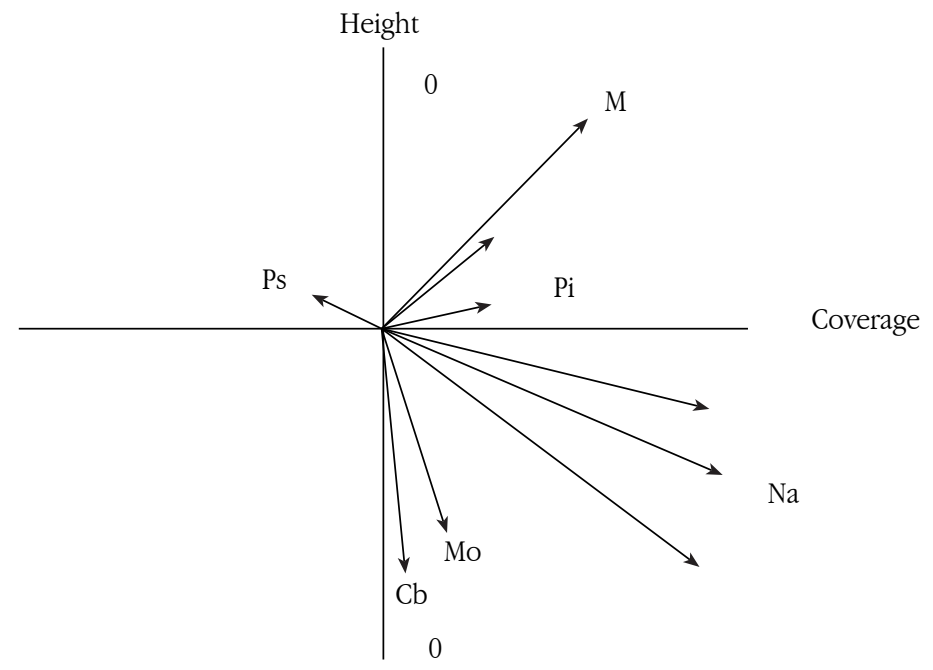

Fig. 2 Canonical Correspondence Analysis 


\section{Discussion}

The list of the bird species recorded and their habits and behaviors observed during the study showed that omnivourous and insectivorous birds formed the largest guild group in the grasslands. The number of individuals of birds of each species in relation to the vegetation type and patchiness showed that short grassland was the most preferred habitat of the birds followed by tall grasslands while covered patches was preferred over open patches. The CCA graph also supports that covered patches was preferred by the birds over open patches. However, the CCA graph in case of the relation between the numbers of individuals to the height of the vegetation (vegetation type) showed as less pronounced relationship which most probably was due to the fact that the different species of birds were associated with different plant/vegetation types according to their habitat requirements.

The high similarity between tall and short grassland bird community along with the fact that the two grasslands also had higher preferences showed that most of the birds recorded during the study were more generalists in nature, capable of inhabiting and utilizing resources in both types of grasslands.

The Kruskall-Walli test showed that there is greater equality of population medians of birds in short grassland compared to tall and wooded grasslands and underlined the greater importance of short grasslands while the Canonical Correspondence Analysis showed that both short and tall grasslands were important for bird communities and underlined the need for maintainenance of the ecological integrity of the area and quality of these habitats for the conservation of grassland birds inside the reserve.

The physical structure of grasslands together with a complex mosaic of various grass species are important factors for determining bird diversity (Baral, 2001). However, the Terai regions of Nepal including Shuklaphanta Wildlife Reserve have undergone massive ecological disturbance, and the loss and degradation of natural and semi-natural grasslands has become the key threat to birds (Baral, 2001). Thus, the park management should focus on the conservation of the current mosaic pattern of both these grasslands leaving larger areas of covered patches during the annual cutting/burning perion for the conservation of the grassland bird communities.

\section{Conclusion}

From the research, it was found that the highest number of species occurred in covered patches of short grassland and the highest species diversity and evenness in covered patches of tall grasslands of Shuklaphanta Wildlife Reserve. The study also found that as the covered patches of both short and tall grasslands were important, it was necessary to maintain the ecological integrity of the area and quality of these habitats for the conservation of grassland birds inside the reserve.

\section{Acknowledgements}

The researcher is indebted to Dr. Thomas Engstrom of USDFW and EMEM award for this study; Prof. Dr. Rejina Maskey of CDES, Dr. Gregorz Mikusinski, Dr. Lars Edenius, and Prof. Dr. Warwiloski of SLU for reviewing the findings; DNPWC for permission to conduct the research and the field monitoring staffs of Shuklaphanta Wildlife Reserve and NTNC/SCP for assistance in field. The researcher is especially thankfult Mr. Binaya Kumar Jha, Mr. Gurudev Chaudhary, Mr. Thaga Chaudhary, Mr. Ramsing Sunar, Mr. Mohan Bhatta, Mr. Devraj Joshi, Captain B. Raut and his platoon for their immense support during field work.

\section{References}

Baral, H.S. (1995). Grassland birds of Nepal. Bird Conservation Nepal, Newsletter, 4, 4.

Baral, H.S. (1997). Suklaphanta: a grassland reserve of international importance. Danphe, 6, 6-8.

Baral, H.S. (2000). Notes on distribution of some grassland birds in Nepal with reference to Suklaphanta. Danphe, 9, 6-7.

Baral, H.S. (2001). Community structure and habitat associations of lowland grassland birds in Nepal (Unpublished doctoral thesis). University of Amsterdam, Netherlands.

BCN (2005). Important bird areas of Nepal: Key sites for conservation. BCN and Birdlife International, Cambridge and Kathmandu.

Birdlife International (2001). Threatened birds of the Asia: the Birdlife International Red Data Book. Birdlife International, Cambridge.

DNPWC (2006). Shuklaphanta management plan. DNPWC, Kathmandu.

Grimeett, R.G., Inskipp, C., Inskipp, T., \& Baral, H. (2003). Birds of Nepal. Birdlife International and Christopher Helm, London.

USDFW (2010). BBIRD Protocol Guidelines. United States Department of Fish and Wildlife, USA. 